\title{
Activation volume details from nonlinear anelastic deformation of a metallic glass
}

Cite as: J. Appl. Phys. 126, 185104 (2019); https://doi.org/10.1063/1.5122973

Submitted: 01 August 2019 . Accepted: 23 October 2019 . Published Online: 11 November 2019

T. J. Lei, and M. Atzmon (D)

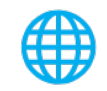

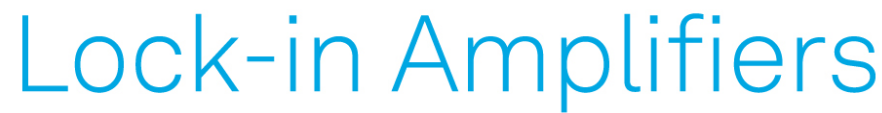

... and more, from DC to $600 \mathrm{MHz}$ Watch 


\title{
Activation volume details from nonlinear anelastic deformation of a metallic glass
}

Cite as: J. Appl. Phys. 126, 185104 (2019); doi: 10.1063/1.5122973

Submitted: 1 August 2019 . Accepted: 23 October 2019 .

Published Online: 11 November 2019

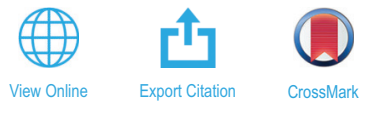

T. J. Lei ${ }^{1}$ and M. Atzmon ${ }^{1,2, a)}$ (iD

\author{
AFFILIATIONS \\ ${ }^{1}$ Department of Materials Science and Engineering, University of Michigan, Ann Arbor, Michigan 48109, USA \\ ${ }^{2}$ Department of Nuclear Engineering and Radiological Sciences, University of Michigan, Ann Arbor, Michigan 48109, USA
}

a) Author to whom correspondence should be addressed: atzmon@umich.edu

\begin{abstract}
At high stress, the viscosity of a metallic glass is non-Newtonian, and therefore the rate of anelastic stress relaxation is not linear in the applied stress. In this regime, one can obtain information on the details of the activation volume that are not accessible in the linear regime. While bending in the nonlinear regime introduces a complicated stress state, it offers great stability for noninstrumented measurements over many orders of magnitude of time. We have developed a method of controlled sample bending to a strain of up to $\sim 0.0155$ for $\mathrm{Al}_{86.8} \mathrm{Ni}_{3.7} \mathrm{Y}_{9.5}$ metallic glass. Significant nonlinearity of the anelastic strain in the stress was observed, which is mainly associated with the largest and slowest shear transformation zones involved not reaching mechanical equilibrium at the end of the constraining period. Combining nonlinear kinetics under constraint and zero bending moment after constraint removal, the volume of the largest shear transformation zones and the transformation shear strain were obtained independently for the inherent state-their most likely values are $4.8 \times 10^{-28} \mathrm{~m}^{3}$ and 0.18 , respectively.
\end{abstract}

Published under license by AIP Publishing. https://doi.org/10.1063/1.5122973

\section{INTRODUCTION}

Metallic glasses (MGs) have drawn considerable attention due to their high strength and elastic limit. ${ }^{1}$ However, they experience flow localization resulting in little macroscopic plasticity, ${ }^{2}$ which limits their structural application. Understanding the deformation mechanism of MGs is necessary to identify ways to improve their plasticity. Unlike for crystalline alloys, knowledge of the microscopic origin of plastic deformation of MGs is incomplete due to their disordered structure. Physical analogs $s^{3,4}$ have shown that macroscopic deformation is accommodated by cooperative shearing of atomic clusters, termed shear transformation zones (STZs). ${ }^{5-8}$

Plastic deformation of metallic glasses involves a large volume fraction of STZs in an activated flow state, for which STZ interactions are complex. In contrast, at small strain, the STZ volume fraction is small, in the dilute limit, so they are isolated. They can be reversed upon removal of external stress due to back stress in the elastic matrix, which leads to anelastic behavior. ${ }^{9}$ In crystalline metals, several mechanisms of anelastic relaxation have been studied. We consider the STZ mechanism to dominate anelasticity in metallic glasses, based on the following points: (a) The Snoek effect of small interstitial solutes ${ }^{10}$ is unlikely in a metallic glass in the absence of, e.g., hydrogen or carbon atoms. (b) Chemical order-disorder effects, ${ }^{11}$ if any, are weak in amorphous metals. (c) Any local jumps of constituent atoms are likely to be STZ mediated. (d) The present experiments involve a single phase and isothermal conditions. ${ }^{11}$

Anelastic deformation in the small-strain regime offers an opportunity to understand plasticity. Ju et al. ${ }^{12}$ performed quasistatic anelastic relaxation measurements of $\mathrm{Al}_{86.8} \mathrm{Ni}_{3.7} \mathrm{Y}_{9.5}$ (at. \%) over a time range spanning seven orders of magnitude. The corresponding relaxation-time spectra were computed, which exhibited distinct peaks, representing an atomically quantized hierarchy of STZs, consisting of 14-21 atoms for the kinetic window observed. The measurements involved small anelastic strain and correspondingly small STZ volume fraction, $1 \%-2 \% .{ }^{12,13}$ Linear dependence of the equilibrated anelastic strain under constraint on the applied stress was observed, which also implied a linear anelastic strain profile across the sample thickness. Consequently, there was no residual stress upon constraint removal.

In the present work, anelastic relaxation is studied at higher strain than in our prior work, such that the viscosity under 
constraint is non-Newtonian, but STZ volume fractions are still small, $\leq 7.2 \%$. Unlike in the linear regime, this approach allows for an independent determination of the STZ volume and transformation strain for the largest activated STZs.

\section{BACKGROUND}

Ju et al. ${ }^{12}$ obtained time-constant spectra for anelastic relaxation for amorphous $\mathrm{Al}_{86.8} \mathrm{Ni}_{3.7} \mathrm{Y}_{9.5}$, which exhibited a set of distinct peaks. They modeled the behavior with a standard linear solid model (Fig. 1) - a spring in series with several Voigt units, each of which represents one peak and STZ size, and contributes additively to the total strain. Analysis of the data, assuming a transformation shear strain of $0.2,{ }^{3,4}$ revealed that each peak corresponded to an STZ size that comprises a discrete number of $\mathrm{Al}$ atoms. A size increment of one atom corresponds to about an order of magnitude larger time constants. Under constraint for $2 \times 10^{6} \mathrm{~s}$ at a fixed strain, all but the largest and slowest STZs, those comprising $n=21$ atoms, essentially reach mechanical equilibrium with each other and the elastic matrix and then track the slow evolution of the $n=21$ STZs. (For longer constraining time, $n=22$ STZs were also activated. $^{13}$ ) After constraint removal, each STZ size evolves independently in this model.

In Ref. 12, the shear strain rate due to STZs indexed with the integer $m$ under an applied shear stress, $\sigma$, was expressed as ${ }^{3}$

$$
\dot{\gamma}_{m}=2 c_{m} \gamma_{o}^{c} v_{G} \exp \left(-\frac{\Delta F_{m}}{k T}\right) \sinh \left(\frac{\sigma \gamma_{o}^{T} \Omega_{m}}{2 k T}\right)
$$

where $m=1, \ldots, 8 . c_{m}$ is the volume fraction occupied by potential $m$-type STZs, where a potential STZ is an atomic cluster that is capable of shear transformation. $\gamma_{o}^{T}$ is the transformation shear strain, and $\gamma_{o}^{c}=[2(4-5 v) /(15(1-v))] \times \gamma_{o}^{T}$ is its value under constraint by the surrounding matrix, with $v$ being Poisson's ratio. $v_{G}$ is the attempt frequency. $\Omega_{m}$ is the volume of $m$-type STZs. The product $\gamma_{o}^{T} \times \Omega_{m}$ is the activation volume for a shear transformation, i.e., the conjugate of the stress. $k$ and $T$ have the usual meaning. For small $\sigma$ values, the hyperbolic sine term can be linearized so that the strain rate is proportional to $\sigma . \Delta F_{m}$ is the activation free energy for shear transformation

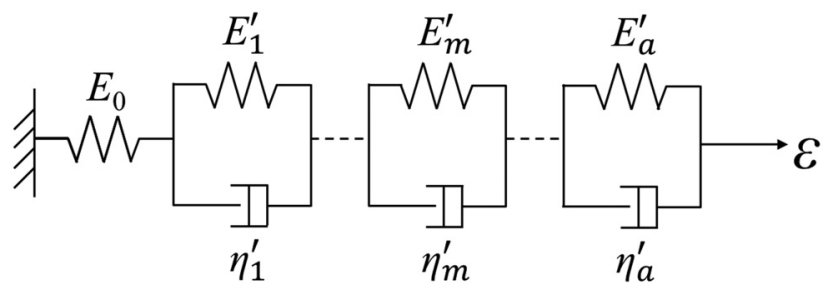

FIG. 1. Schematic illustration of the standard linear solid model employed-a spring with Young's modulus $E_{0}$ in series with Voigt units, each of which represents one STZ size. $E_{m}^{\prime}$ and $\eta_{m}^{\prime}$ are the effective Young's modulus and effective viscosity, respectively, of $m$-type STZs, where $m=1-8$ for the range of time values in the experiment. ${ }^{12}$ Reproduced with permission from Ju et al., J. Appl. Phys. 109, 053522 (2011). Copyright 2011 AIP Publishing LLC. of $m$-type STZs, ${ }^{14}$

$$
\Delta F_{m}=\left[\left(\frac{7-5 v}{30(1-v)}+\frac{2(1+v)}{9(1-v)} \bar{\beta}^{2}\right) \times \gamma_{o}^{T}+\frac{1}{2} \times \frac{\overline{\sigma_{S T Z}}}{\mu}\right] \mu \gamma_{o}^{T} \Omega_{m}
$$

$\bar{\beta}^{2}$ is the dilatancy factor and approximately equal to $1, \mu$ is the shear modulus and expressed as $E_{0} /[2(1+v)]$, and $E_{0}$ is Young's modulus. $\overline{\sigma_{S T Z}}$ is the shear resistance of an STZ if it were not embedded in the matrix. The three terms in Eq. (2) correspond, respectively, to the (a) shear strain energy, (b) dilatation strain energy, and (c) shear energy of the STZ if it were not embedded in the matrix. ${ }^{15}$ The parameters in Eqs. (1) and (2), their values, and sources are summarized in Table I and the Nomenclature.

In the linear, i.e., Newtonian regime, the product $\left(\gamma_{0}^{T}\right)^{2} \times \Omega_{m}$ can be determined, but not each factor independently. An estimated value of $\gamma_{0}^{T}=0.2$, based on physical analogs, ${ }^{3,4}$ was used to obtain $\Omega_{m}, m=1, \ldots, 8$ in Refs. 12 and 13 . These values were spaced by a single atomic volume. Each $\Omega_{m}$ value is associated with an integer multiple of the atomic value of $\mathrm{Al}: \Omega_{n}=n \times \Omega_{\mathrm{Al}}$, where $n=13+m$. The range of $n$ values, $14 \leq n \leq 21$, is determined by the range of experimental time scales. In order to determine $\gamma_{0}^{T}$ and $\Omega_{n=21}$ independently, it is necessary to perform measurements at higher stress, in the non-Newtonian regime, where Eq. (1) is not linear in $\sigma$. Such an approach was reported for large tensile strains, up to 0.08 , using strain-rate jumps and assuming a single STZ size. ${ }^{14}$ The present work involves anelasticity measurements in the non-Newtonian regime, but in contrast to Ref. 14, we employ a maximum bending strain of 0.0155 , with maximum anelastic shear strain of 0.0060 , such that the volume fraction occupied by STZs is still small, $\leq 7.2 \%$. Therefore, STZ properties are obtained for an inherent state, i.e., a local minimum of the energy landscape. While uniaxial geometry offers zero residual stress and far simpler analysis, experiments in bending geometry, not being instrumented, allow for high precision in a wide dynamic range of time, $\sim 10^{2}-3.0 \times 10^{7} \mathrm{~s}$ for the present work. Using the constitutive law [Eq. (1)] for $n=21$ STZs and zero-bending-moment condition after constraint removal and complete reversal of STZs with $n<21$, strain data for $\mathrm{Al}_{86.8} \mathrm{Ni}_{3.7} \mathrm{Y}_{9.5}$ are analyzed, accounting for residual stresses. The volume of the largest and slowest STZ size, for a constraining period of $2 \times 10^{6} \mathrm{~s}, \Omega_{n=21}$, and the transformation shear strain are obtained independently.

\section{EXPERIMENTAL DETAILS}

Amorphous $\mathrm{Al}_{86.8} \mathrm{Ni}_{3.7} \mathrm{Y}_{9.5}$ (at. \%) ribbons, $22 \mu \mathrm{m}$ thick and $1 \mathrm{~mm}$ wide, were produced by single-wheel melt-spinning using a $\mathrm{Cr}$-coated $\mathrm{Cu}$ wheel at a tangential velocity of $40 \mathrm{~m} / \mathrm{s}$ in vacuum. In previous bend relaxation measurements, ${ }^{12}$ samples were constrained by wrapping around mandrels with radii ranging from 0.35 to $0.49 \mathrm{~cm}$, corresponding to equilibrium elastic bending-strain values from 0.00158 to 0.00303 at the surface. To obtain higher bending strain, up to 0.0155 , a constraining method was developed for smaller radii, as illustrated in Fig. 2(a): a sample is placed between a mandrel (radius 0.09 or $0.11 \mathrm{~cm}$ ) and neoprene block. A machined device is used to press the mandrel until the two ends of the sample just touch each other, so that a well-characterized 
TABLE I. Parameter definitions in the expression of shear strain rate [Eq. (1)] and activation free energy [Eq. (2)].

\begin{tabular}{|c|c|c|}
\hline Symbol & Physical meaning & Value/Expression \\
\hline$c_{m}$ & Volume fraction occupied by potential $m$-type STZs & $\begin{array}{l}\text { Area of corresponding spectrum peak from experiments } \\
\text { (Refs. } 12 \text { and 13) }\end{array}$ \\
\hline$m$ & Index of spectrum peaks & $1, \ldots, 8$ \\
\hline & & $\begin{aligned}{ }^{*} \text { Note: } n= & 13+m=\text { number of atoms in STZ is used as } \\
& \text { a subscript in Eq. (9) and below it }\end{aligned}$ \\
\hline$\gamma_{o}^{c}$ & $\begin{array}{c}\text { Transformation shear strain under constraint by } \\
\text { surrounding matrix }\end{array}$ & $\gamma_{o}^{c}=[2(4-5 v) /(15(1-v))] \times \gamma_{o}^{T}$ \\
\hline$v_{G}$ & Attempt frequency & $10^{13} \mathrm{~s}^{-1}$ (Ref. 16) \\
\hline$T$ & Temperature & $295.15 \mathrm{~K}$ \\
\hline$\Delta F_{m}$ & Activation barrier associated with $m$-type STZs & Eq. (2) \\
\hline$\sigma$ & Applied shear stress & Expressed in Eqs. (8) and (16) \\
\hline$\Omega_{m}$ & Volume of $m$-type STZs & To be determined \\
\hline $\bar{\beta}^{2}$ & Dilatancy factor & $\sim 1$ (Ref. 14) \\
\hline$\overline{\sigma_{S T Z}}$ & Peak interatomic shear stress between atoms in a regular lattice & $\overline{\sigma_{S T Z}} / \mu=0.025$ (Ref. 17) \\
\hline$v$ & Poisson’s ratio & 0.35 (Ref. 18) \\
\hline$E_{0}$ & Young's modulus of the matrix & 48.2 GPa (Ref. 19) \\
\hline$\mu$ & Shear modulus of the matrix & $E_{0} /[2(1+v)]$ \\
\hline$\gamma_{o}^{T}$ & $\begin{array}{c}\text { Transformation shear strain in the absence of constraint by the } \\
\text { surrounding matrix }\end{array}$ & To be determined \\
\hline
\end{tabular}

geometry is obtained. A peephole on the side surface of the machined device is used to observe the two touching ends of the sample during constraining [Fig. 2(b)]. Because of the low modulus of the neoprene, the pressure on the sample is negligible compared to the bending stress. A lubricant was applied between the sample and neoprene to minimize friction. In contrast to the constraining configuration in Ref. 12, in which the entire sample was under constraint, in the present work, only a small section is under constraint, with the two free ends allowing for a reliable determination of the radius of curvature. The detailed constraining geometry is shown in Fig. 3(a). $1 \mathrm{~cm}$ long samples were used, and all measurements were performed at room temperature.

As in Ref. 12, samples were constrained for $t_{c}^{\text {total }}=2 \times 10^{6} \mathrm{~s}$ and then relaxed unconstrained for up to $3 \times 10^{7} \mathrm{~s}$. The evolution

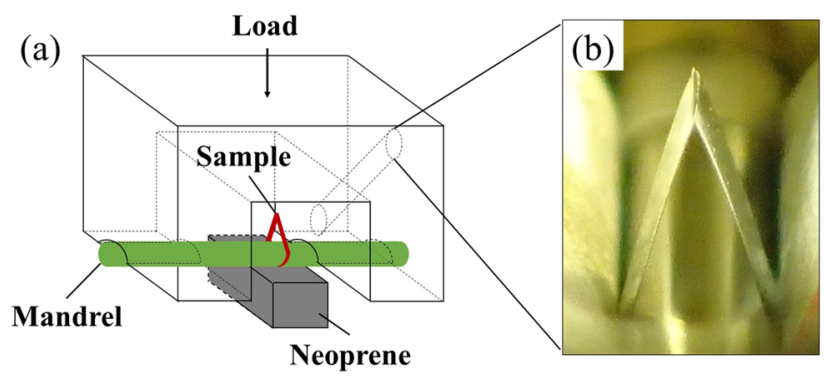

FIG. 2. (a) Schematic illustration of the constraining method-the sample is placed between a mandrel and neoprene block, and a machined device is used to press the mandrel until the two free ends of the sample just touch each other. (b) Photograph showing the two touching ends of a sample under constraint. in the angle between the two ends during unconstrained relaxation [Fig. 3(b)] was recorded with a digital camera and used to determine the curvature of the previously bent section. A stage micrometer was used for calibration, and the optical axis of the camera was aligned perpendicular to the sample stage.

The total constraining strain at a distance $y$ from the sample midplane is

$$
\varepsilon_{\text {constr }}(y)=y \times\left(1 / R-1 / r_{0}\right),
$$

where $R$ is the mandrel radius and $r_{0}$ is the initial radius of curvature for the sample before constraining. At the end of the constraining period, lasting $t_{c}^{\text {total }}, \varepsilon_{\text {constr }}(y)$ includes both an elastic and
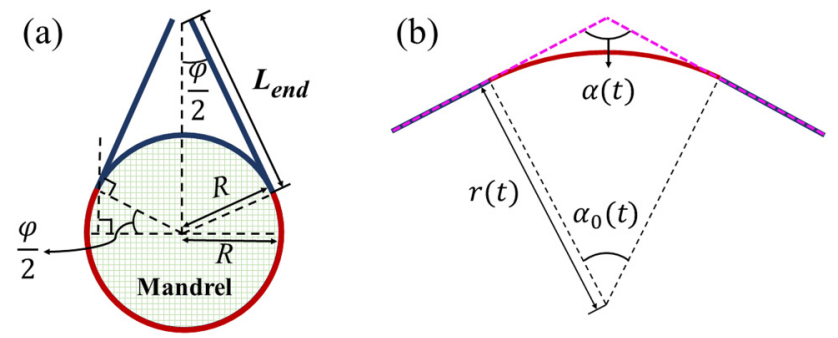

FIG. 3. Sample geometry (a) under constraint and (b) during unconstrained relaxation (not to scale). $\alpha(t)$ is used to determine the evolution of the curvature of the previously bent section during unconstrained relaxation. The length of the constrained section (red) is equal to $(\pi+\varphi) \times(R+d / 2)$, where $R$ is the mandrel radius and $d$ is the sample thickness. Dashed lines are fits to the unconstrained ends. The small curvature of the free ends is neglected in these plots. 
an anelastic component. We can determine apparent strain values, based on linear variation with $y$. The apparent elastic strain is

$$
\varepsilon_{e l, \text { constr }}^{a p p}\left(y, t_{c}^{\text {total }}\right)=y \times[1 / R-1 / r(0)]
$$

where $r(t)$ is the radius of curvature of the previously constrained section at time $t$ after constraint removal. The apparent anelastic strain is

$$
\varepsilon_{a n, c o n s t r}^{a p p}\left(y, t_{c}^{\text {total }}\right)=y \times\left[1 / r(0)-1 / r_{0}\right]
$$

Note that unlike the elastic strain, the apparent anelastic strain at the end of the constraining period is equal to its value at $t=0$ after constraint removal, $\varepsilon_{a n}^{a p p}(y, t=0)$.

Substitution of $r(t)$ for $r(0)$ in Eq. (5) provides the apparent anelastic strain at time $t$ after constraint removal. In the case of Newtonian behavior, when the strain profile across the sample thickness is linear, the expressions for the apparent strains are equal to the actual values. However, in the nonlinear regime, the anelastic strain is superlinear close to the surface. As a result, there is residual stress near each surface in the unconstrained state, with signs opposite of those under constraint. $\varepsilon_{a n}^{a p p}(d / 2, t), d$ being the sample thickness, is then lower than the actual anelastic strain at the surface.

While it would be challenging to directly measure the radius of curvature of the small constrained section during unconstrained relaxation, the well-characterized geometry (Fig. 3) allows for a reliable determination of $r(t)$ from the angle between two fit lines to the free ends [dashed lines in Fig. 3(b)]. For different mandrel radii used, the maximum constraining strain ranges from 0.0079 to 0.0155 , below the yield point $(\sim 0.02){ }^{20}$ This was verified by the observation that constraining for a short duration did not lead to permanent deformation. The estimated volume fraction occupied by STZs, based on the anelastic strain, is between $3 \%$ and $7.2 \%$, still in the dilute limit.

It was shown in Ref. 12 that the time constant for anelastic recovery of the largest active STZs (consisting of $21 \mathrm{Al}$ atoms) is $\tau_{21}=1.25 \times 10^{7} \mathrm{~s}$, significantly longer than the total constraining time $\left(t_{c}^{\text {total }}=2 \times 10^{6} \mathrm{~s}\right)$. All other $\tau_{i}, i \leq 20$, are smaller than $10^{6} \mathrm{~s} .{ }^{12}$ It follows that all but the $n=21$ STZs nearly equilibrate by the end of the constraining period. Since nonlinearity affects the kinetics but not the mechanical equilibrium state, it therefore only affects the contribution of $n=21 \mathrm{STZs}$, as these do not equilibrate during the constraining time. In order to isolate this contribution, the apparent anelastic strain after $t=4 \times 10^{6} \mathrm{~s}$ unconstrained relaxation is shown in Fig. 4 as a function of the apparent elastic strain at the end of the constraining period, both computed for the sample surface. The contribution of STZs with $n \leq 20$ atoms to the anelastic strain is negligible at this point $\left(t=4 \times 10^{6} \mathrm{~s}\right)$, since these have essentially been fully reversed. The five small-strain data points in Fig. 4, which lie on a straight line, are taken from Ref. 12. Significant deviation from linearity is observed at high strain and stress. The decrease of the anelastic strain due to $n=21 \mathrm{STZ}$ from its value at the end of the constraining period, about $27 \%$ during unconstrained relaxation for $4 \times 10^{6} \mathrm{~s}$, is accounted for in the analysis.

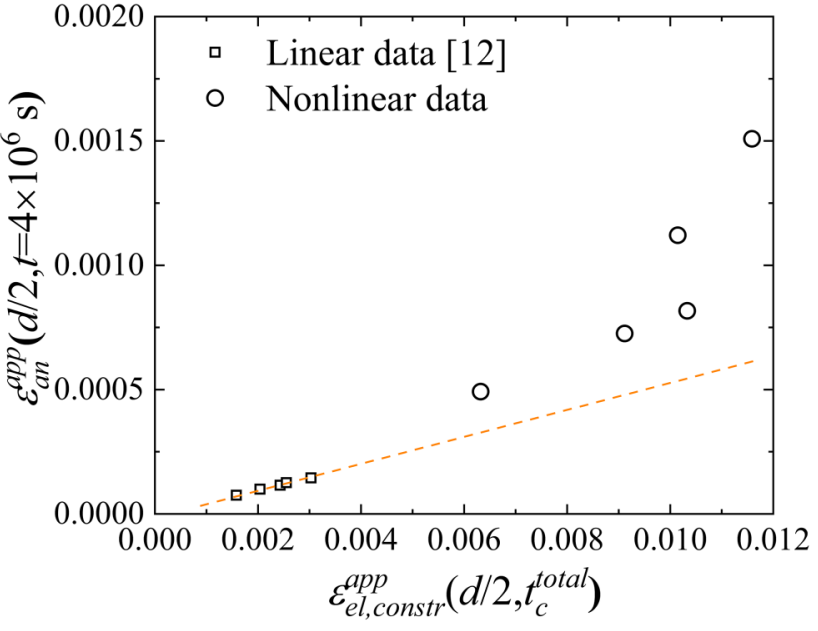

FIG. 4. Apparent anelastic strain after unconstrained relaxation for $t=4 \times 10^{6} \mathrm{~s}$ as a function of the apparent elastic strain at the end of the constraining period for varying constraining radii. Both are computed for the sample surface. Each symbol represents one sample. Deviation from linearity occurs at high strain. The dashed line is a fit to the linear portion.

\section{OVERVIEW OF THE DATA ANALYSIS}

"Apparent" strain values below are those determined from curvature by using linear variation across the sample. The term "actual" is used to distinguish strain and stress values from their apparent values. The Nomenclature contains a summary of the notation used below.

1. Applying the condition of zero total bending moment after constraint removal, a relationship between the apparent anelastic strain at the sample surface at $t=4 \times 10^{6} \mathrm{~s}$ after constraint removal, $\boldsymbol{\varepsilon}_{a n}^{a p p}\left(\boldsymbol{d} / 2, \boldsymbol{t}=4 \times 10^{6} \mathrm{~s}\right)$, and the position-dependent anelastic strain due to $n=21$ STZs at the end of the constraining period, $\varepsilon_{21, \text { constr }}\left(y, t_{c}^{\text {total }}\right)$, is obtained.

2. $\boldsymbol{\varepsilon}_{\boldsymbol{a} \boldsymbol{n}}^{a p p}\left(\boldsymbol{d} / 2, \boldsymbol{t}=4 \times 10^{6} \mathrm{~s}\right)$ is directly determined from curvature measurements [Eq. (5)]. An expression for $\varepsilon_{21, \text { constr }}\left(y, t_{c}^{\text {total }}\right)$, in terms of the apparent elastic strain at the sample surface at the end of the constraining period, $\varepsilon_{e l, c o n s t r}^{a p p}\left(d / 2, t_{c}^{\text {total }}\right)$, is obtained from time integration of the position- and time-dependent macroscopic shear strain rate due to $n=21$ STZs under constraint, $\dot{\gamma}_{21, \text { constr }}\left(y, t_{c}\right)$. Approximations used in this step are detailed below.

3. Substituting the expression for $\varepsilon_{21, \text { constr }}\left(y, t_{c}^{\text {total }}\right)$ into the relationship between $\boldsymbol{\varepsilon}_{a n}^{a p p}\left(\boldsymbol{d} / 2, \boldsymbol{t}=4 \times 10^{6} \mathrm{~s}\right)$ and $\varepsilon_{21, \text { constr }}\left(y, t_{c}^{\text {total }}\right)$, obtained in step 1 above, yields a fitting equation for $\boldsymbol{\varepsilon}_{a n}^{a p p}\left(\boldsymbol{d} / 2, \boldsymbol{t}=4 \times 10^{6} \mathrm{~s}\right)$ vs $\boldsymbol{\varepsilon}_{\text {el,constr }}^{a p p}\left(\boldsymbol{d} / 2, \boldsymbol{t}_{\boldsymbol{c}}^{\text {total }}\right)$. This equation contains two fitting parameters: the transformation shear strain, $\gamma_{0}^{T}$, and the volume of $n=21 \mathrm{STZ}, \Omega_{21}$.

4. A simultaneous two-parameter fit is performed on all data. The linear portion, which has smaller error, is not fitted as well as with a separate linear fit. A revised two-step fit is performed as follows: first, the linear regime is fitted, which yields the value of $\left(\gamma_{0}^{T}\right)^{2} \times \Omega_{21}$ with small random error. This 
value is then used as a constraint for the entire data set to obtain $\Omega_{21}$ and $\gamma_{0}^{T}$.

\section{ANALYSIS DETAILS}

We first note that for the presently used bending-strain values $\leq 0.0155$, nonlinear elastic behavior ${ }^{21}$ is likely to be minimal, especially since the long-range elastic field of an STZ dominates $\Delta F$ in Eq. (2). Since nonlinearity is observed in Fig. 4, nonlinear kinetics [Eq. (1)] now will be used. After constraint removal, the total bending moment is zero,

$$
M(t=0)=\int_{-\frac{d}{2}}^{\frac{d}{2}}\left[\sigma_{\text {constr }}\left(y, t_{c}^{\text {total }}\right)-\sigma_{u}(y, t=0)\right] \times y d y=0,
$$

where $\sigma_{\text {constr }}\left(y, t_{c}^{\text {total }}\right)$ is the applied stress at a distance $y$ from the sample midplane at the end of the constraining period. $\sigma_{u}(y, t=0)$ is the unloading stress immediately upon constraint removal, which varies linearly across the sample thickness,

$$
\sigma_{u}(y, t=0)=y \times\left[\frac{1}{R}-\frac{1}{r(0)}\right] \times \frac{E_{0}}{2\left(1-v^{2}\right)} .
$$

The position-dependent applied stress at the end of the constraining period is

$$
\sigma_{\text {constr }}\left(y, t_{c}^{\text {total }}\right)=\left[\varepsilon_{\text {constr }}(y)-\varepsilon_{\text {an,constr }}\left(y, t_{c}^{\text {total }}\right)\right] \times \frac{E_{0}}{2\left(1-v^{2}\right)},
$$

where $\varepsilon_{\text {constr }}(y)$ is the position-dependent total constraining strain, $y \times\left[1 / R-1 / r_{0}\right] . \varepsilon_{a n, c o n s t r}\left(y, t_{c}^{t o t a l}\right)$ is the sum of the positiondependent anelastic strain due to all active STZ sizes at the end of the constraining period. It consists of contributions: (a) due to STZs comprising $n$ atoms, $\varepsilon_{n, \text { constr }}^{0}\left(y, t_{c}^{\text {total }}\right), n=14-20$, which reached mechanical equilibrium during constraining, and (b) due to $n=21$ STZs, $\varepsilon_{21, \text { constr }}\left(y, t_{c}^{\text {total }}\right)$-these did not reach mechanical equilibrium. Therefore, $\varepsilon_{\text {an,constr }}\left(y, t_{c}^{\text {total }}\right)$ is expressed as

$$
\begin{aligned}
\varepsilon_{a n, \mathrm{constr}}\left(y, t_{c}^{\text {total }}\right) & =\sum_{n=14}^{20} \varepsilon_{n, \text { constr }}^{0}\left(y, t_{c}^{\text {total }}\right)+\varepsilon_{21, \text { constr }}\left(y, t_{c}^{\text {total }}\right) \\
& =\sum_{n=14}^{20} c_{n} \times \varepsilon_{e l, c o n s t r}\left(y, t_{c}^{\text {total }}\right)+\varepsilon_{21, \text { constr }}\left(y, t_{c}^{\text {total }}\right) .
\end{aligned}
$$

$\varepsilon_{n, \text { constr }}^{0}\left(y, t_{c}^{\text {total }}\right)$ equals $c_{n} \times \varepsilon_{e l, \text { constr }}\left(y, t_{c}^{\text {total }}\right),{ }^{12}$ where $c_{n}$ is the volume fraction occupied by potential STZs comprising $n$ atoms, and $\varepsilon_{e l, \text { constr }}\left(y, t_{c}^{\text {total }}\right)$ is the position-dependent elastic strain at the end of the constraining period. The values of $c_{n}$ were obtained in Refs. 12 and 13.
Substituting Eqs. (7)-(9) into Eq. (6), and since $\sigma_{\text {constr }}\left(y, t_{c}^{\text {total }}\right)$ and $\sigma_{u}(y, t=0)$ are antisymmetric, Eq. (6) immediately after constraint removal becomes

$$
\begin{aligned}
\int_{0}^{\frac{d}{2}} y \times\left[\frac{1}{r(0)}-\frac{1}{r_{0}}\right] \times y d y= & \int_{0}^{\frac{d}{2}} \sum_{n=14}^{20} c_{n} \times \varepsilon_{e l, \text { constr }}\left(y, t_{c}^{\text {total }}\right) \times y d y \\
& +\int_{0}^{\frac{d}{2}} \varepsilon_{21, \text { constr }}\left(y, t_{c}^{\text {total }}\right) \times y d y .
\end{aligned}
$$

After $t=4 \times 10^{6} \mathrm{~s}$ of unconstrained relaxation, the contribution of STZs with $14 \leq n \leq 20$ vanishes, and with $\tau_{21}=1.25 \times 10^{7} \mathrm{~s}$, ${ }^{12}$ the anelastic strain due to $n=21$ decreases by a factor of 0.73 . Consequently, at $t=4 \times 10^{6} \mathrm{~s}$, the zero-moment condition becomes

$$
\begin{aligned}
\boldsymbol{\varepsilon}_{a n}^{a p p}\left(\boldsymbol{d} / 2, \boldsymbol{t}=4 \times 10^{6} \mathrm{~s}\right)= & 0.73 \times \frac{12}{d^{2}} \\
& \times \int_{0}^{\frac{d}{2}} \varepsilon_{21, \text { constr }}\left(y, t_{c}^{\text {total }}\right) \times y d y .
\end{aligned}
$$

The position-dependent anelastic bending strain due to $n=21$ STZs at the end of the constraining period is

$$
\varepsilon_{21, \text { constr }}\left(y, t_{c}^{\text {total }}\right)=(1-v) \times \int_{0}^{t_{c}^{\text {total }}} \dot{\gamma}_{21, \text { constr }}\left(y, t_{c}\right) d t_{c},
$$

where $(1-v)$ is the ratio of bending to shear strain. $\dot{\gamma}_{21, \text { constr }}\left(y, t_{c}\right)$ is the actual position-dependent macroscopic shear strain rate due to $n=21$ STZs after time $t_{c}$ under constraint. Similar to the total anelastic strain, $\varepsilon_{21, \text { constr }}\left(y, t_{c}^{\text {total }}\right)$ is also equal to the anelastic strain due to $n=21$ STZs immediately following constraint removal, $\varepsilon_{21}(y, t=0)$. Using Eq. (1) with $n=21$ corresponding to $m=8$, the actual position-dependent macroscopic shear strain rate due to $n=21$ STZs as function of time under constraint is

$$
\dot{\gamma}_{21, \text { constr }}\left(y, t_{c}\right)=2 c_{21} \gamma_{0}^{c} v_{G} \exp \left[-\frac{\Delta F_{21}}{k T}\right] \sinh \left[\frac{\sigma_{\text {constr }}\left(y, t_{c}\right) \gamma_{0}^{T} \Omega_{21}}{2 k T}\right] .
$$

The last term in Eq. (2), the shear resistance of an STZ isolated from the matrix, can be neglected, ${ }^{14}$ yielding

$$
\Delta F_{21}=\left[\frac{(7-5 v)}{30(1-v)}+\frac{2(1+v)}{9(1-v)} \times \bar{\beta}^{2}\right] \mu\left(\gamma_{0}^{T}\right)^{2} \Omega_{21}
$$

Substituting Eq. (14) into Eq. (13), the position-dependent strain rate due to $n=21 \mathrm{STZs}$ under constraint lasting $t_{c}$, $0 \leq t_{c} \leq t_{c}^{\text {total }}$, becomes

$$
\dot{\gamma}_{21, \text { constr }}\left(y, t_{c}\right)=2 c_{21} \gamma_{0}^{c} v_{G} \times \exp \left[-\left[\frac{(7-5 v)}{30(1-v)}+\frac{2(1+v)}{9(1-v)} \times \bar{\beta}^{2}\right] \times \frac{\mu\left(\gamma_{0}^{T}\right)^{2} \Omega_{21}}{k T}\right] \times \sinh \left[\frac{\sigma_{c o n s t r}\left(y, t_{c}\right) \gamma_{0}^{T} \Omega_{21}}{2 k T}\right]
$$


In Eq. (15), the only parameter expected to change significantly with time is the applied stress $\sigma_{\text {constr }}\left(y, t_{c}\right)$, since the constraint imposes a fixed total strain. However, since the apparent elastic strain at the sample surface at the end of the constraining period remains within $\sim 25 \%$ of its value at the beginning, $\sigma_{\text {constr }}\left(y, t_{c}\right)$ will be approximated by the latter value, $\sigma_{\text {constr }}\left(y, t_{c}\right) \approx \sigma_{\text {constr }}\left(y, t_{c}^{\text {total }}\right)$. Consequently, $\dot{\gamma}_{21, \text { constr }}\left(y, t_{c}\right)$ is approximated as its value at $t_{c}^{\text {total }}$. This is one of the two approximations used to estimate Eq. (15). The second approximation is based on the apparent elastic strain, as detailed below.

The position-dependent applied stress at the end of the constraining period, $\sigma_{\text {constr }}\left(y, t_{c}^{\text {total }}\right)$, is proportional to the actual position-dependent elastic strain at that point, $\varepsilon_{\text {el,constr }}\left(y, t_{c}^{\text {total }}\right)$ $=\varepsilon_{\text {constr }}(y)-\varepsilon_{\text {an,constr }}\left(y, t_{c}^{\text {total }}\right)$. It will be approximated by its apparent value, Eq. (4), since $\boldsymbol{\varepsilon}_{\boldsymbol{e l , c o n s t r}}^{\text {app }}\left(\boldsymbol{d} / 2, \boldsymbol{t}_{\boldsymbol{c}}^{\text {total }}\right)$ is within $\sim 25 \%$ of $\varepsilon_{\text {constr }}(d / 2)$. Therefore,

$$
\sigma_{\text {constr }}\left(y, t_{c}^{\text {total }}\right) \approx \varepsilon_{e l, c o n s t r}^{a p p}\left(y, t_{c}^{\text {total }}\right) \times \frac{E_{0}}{2\left(1-v^{2}\right)} .
$$

Substituting the values of all known parameters, listed above, and Eq. (16) into Eq. (15) yields the approximate positiondependent macroscopic shear strain rate due to $n=21$ STZs at the end of the constraining period,

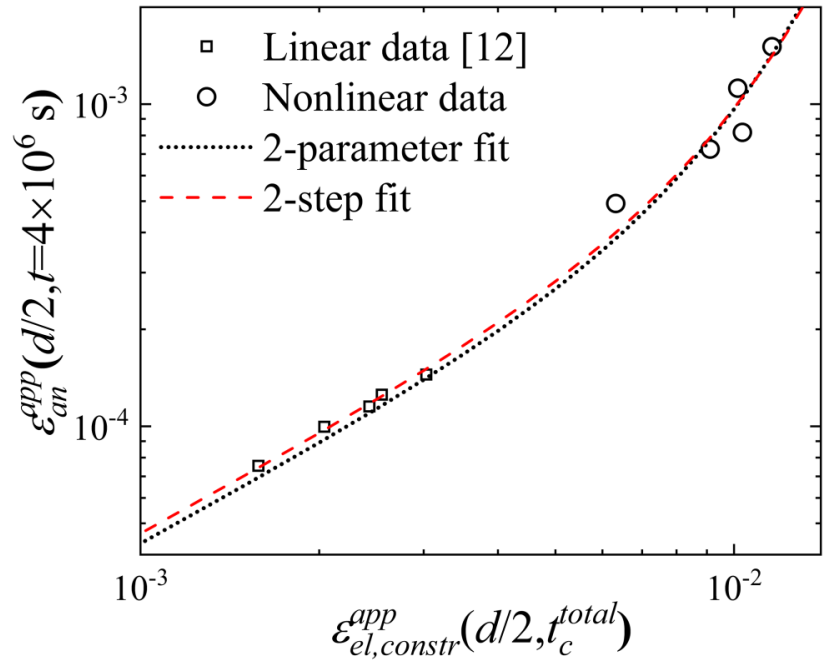

FIG. 5. Data of Fig. 4 on a log-log scale. Comparison between the twoparameter fit (dotted line) and two-step fit (dashed line). The latter yields a better fit for the small-strain data than the former.

$$
\dot{\gamma}_{21, \text { constr }}^{\text {appr }}\left(y, t_{c}^{\text {total }}\right)=1.2 \times 10^{12} \times \gamma_{0}^{T} \times \exp \left[-3.20 \times 10^{30} \times\left(\gamma_{0}^{T}\right)^{2} \times \Omega_{21}\right] \times \sinh \left[3.37 \times 10^{30} \times \varepsilon_{\text {el,constr }}^{\text {app }}\left(y, t_{c}^{\text {total }}\right) \times \gamma_{0}^{T} \times \Omega_{21}\right]
$$

with $\Omega_{21}$ in $\mathrm{m}^{3}$ here and below. Substituting Eq. (17) as an approximation of $\dot{\gamma}_{21, \text { constr }}\left(y, t_{c}\right)$ into Eq. (12) yields

$$
\varepsilon_{21, \text { constr }}\left(y, t_{c}^{\text {total }}\right)=2 \times 10^{6} \times 0.65 \times 1.2 \times 10^{12} \times \gamma_{0}^{T} \times \exp \left[-3.20 \times 10^{30} \times\left(\gamma_{0}^{T}\right)^{2} \times \Omega_{21}\right] \times \sinh \left[3.37 \times 10^{30} \times \varepsilon_{e l, \text { constr }}^{a p p}\left(y, t_{c}^{\text {total }}\right) \times \gamma_{0}^{T} \times \Omega_{21}\right]
$$

Substituting Eq. (18) into Eq. (11) results in

$\boldsymbol{\varepsilon}_{\boldsymbol{a n}}^{\boldsymbol{a p p}}\left(\boldsymbol{d} / 2, \boldsymbol{t}=4 \times 10^{6} \mathbf{s}\right)=3.42 \times 10^{18} \times \gamma_{0}^{T} \times \exp \left[-3.20 \times 10^{30} \times\left(\gamma_{0}^{T}\right)^{2} \times \Omega_{21}\right] \times \int_{0}^{1} \sinh \left[3.37 \times 10^{30} \times \boldsymbol{\varepsilon}_{\text {el, constr }}^{\text {app }}\left(\boldsymbol{d} / 2, \boldsymbol{t}_{\boldsymbol{c}}^{\text {total }}\right) \times z \times \gamma_{0}^{T} \times \Omega_{21}\right] z d z$,

where $z=2 y / d$. Equation (19) is the fitting equation for measured values of $\boldsymbol{\varepsilon}_{a n}^{a p p}\left(\boldsymbol{d} / 2, \boldsymbol{t}=4 \times 10^{6} \mathrm{~s}\right)$ as a function of $\boldsymbol{\varepsilon}_{\text {el,constr }}^{\text {app }}\left(\boldsymbol{d} / 2, \boldsymbol{t}_{\boldsymbol{c}}^{\text {total }}\right)$ (Fig. 4), with $\gamma_{0}^{T}$ and $\Omega_{21}$ being the fitting parameters. A Taylor series up to the $11^{\text {th }}$ order (six terms) is used as a good approximation of the hyperbolic sine function.

A two-parameter fit is performed on the entire range of data simultaneously, with equal weight to all points. It is shown in Fig. 5, which displays the data of Fig. 4 on logarithmic scales. The fit yields $\gamma_{0}^{T}=0.17$ and $\Omega_{21}=5.2 \times 10^{-28} \mathrm{~m}^{3}$ with a R-squared value of 0.982 . The fit sensitivity to each $\gamma_{0}^{T}$ and $\Omega_{21}$ is determined by fixing one at different values and using the other as a single fitting parameter. This yields estimated random errors in $\gamma_{0}^{T}$ and $\Omega_{21}$ of $\pm 3 \%$ and $\pm 6 \%$, respectively. The main approximation has been to express the applied stress that drives anelastic deformation as linearly varying in $y$ and constant in time. An attempt at fit improvement was made by using the resulting strain distribution to update the applied stress and iterate to obtain a revised fit. The iteration yields a very small change in $\gamma_{0}^{T}$ and $\Omega_{21}$. Moreover, it leads to a slightly worse fit of the (low-scatter) linear portion and is not considered useful. We conclude that the scatter of the data limits any further improvement in the two-parameter fit.

In Fig. 5, it is apparent that the two-parameter fit deviates from the linear portion of the data. Since this portion is more reliable, a revised two-step fit is now employed. For the linear regime, 
the hyperbolic sine term in Eq. (19) is linearized,

$$
\begin{aligned}
\boldsymbol{\varepsilon}_{\text {an }}^{\text {app }}\left(\boldsymbol{d} / 2, \boldsymbol{t}=4 \times 10^{6} \mathrm{~s}\right)= & 3.84 \times 10^{48} \times\left(\gamma_{0}^{T}\right)^{2} \times \Omega_{21} \\
& \times \exp \left[-3.20 \times 10^{30} \times\left(\gamma_{0}^{T}\right)^{2} \times \Omega_{21}\right] \\
& \times \boldsymbol{\varepsilon}_{\text {el, } \text { constr }}^{\text {app }}\left(\boldsymbol{d} / 2, \boldsymbol{t}_{\boldsymbol{c}}^{\text {total }}\right) .
\end{aligned}
$$

Fitting Eq. (20) to the small-strain data yields a slope of 0.0482, which, when substituted into Eq. (20), yields

$$
\left(\gamma_{0}^{T}\right)^{2} \times \Omega_{21}=1.517 \times 10^{-29} \mathrm{~m}^{3},
$$

with a random error of only a small fraction of a percent because this term appears in the exponent in Eq. (19). Substituting Eq. (21) into Eq. (19) and fitting the entire range of data yields $\gamma_{0}^{T}=0.18$ and $\Omega_{21}=4.8 \times 10^{-28} \mathrm{~m}^{3}$ with random errors of $1.5 \%$ and $3 \%$, respectively. Since the error in $\left(\gamma_{0}^{T}\right)^{2} \times \Omega_{21}$ is much smaller, these two errors are strongly correlated. The R-squared value of the fit is 0.982 , which equals that for the two-parameter fit.

The implication of these new results, $\gamma_{0}^{T}=0.18$ and $\Omega_{21}=4.8 \times 10^{-28} \mathrm{~m}^{3}$ ( $\sim 29 \mathrm{Al}$ atoms $)$, for Ref. 12 are now discussed. If one assumes $\gamma_{0}^{T}=0.18$ to be independent of STZ size, it can be used to recalculate the results of Ref. 12. While the previous quantized hierarchy still stands, the present numerical values result in a volume increment of $2.08 \times 10^{-29} \mathrm{~m}^{3}$, in contrast to Ref. 12, in which it was fortuitously close to the volume of an $\mathrm{Al}$ atom $\left(V_{\mathrm{Al}}=1.66 \times 10^{-29} \mathrm{~m}^{3}\right)$. This possibly highlights the limitation of the model of Fig. 1 and constitutive law of Refs. 12 and 14 [Eq. (1)]. The present results provide a confirmation of the magnitude of $\gamma_{0}^{T}$, for which an approximate value of 0.2 , obtained from physical analogs, ${ }^{3,4}$ was used in our prior work.

Interestingly, despite the lower strain and corresponding STZ volume fraction in the present work, the activation volume we obtain, $\gamma_{0}^{T} \times \Omega_{21}=8.6 \times 10^{-29} \mathrm{~m}^{3}$, is similar to values obtained from creep in $\mathrm{Pd}_{80} \mathrm{Si}_{20},{ }^{14} 10.5 \times 10^{-29} \mathrm{~m}^{3}$, and from viscosity measurements in undercooled melts, $8 \times 10^{-29}-1.9 \times 10^{-28} \mathrm{~m}^{3} .{ }^{22}$ Because of the different assumptions in Ref. 22, its STZ volume values are about a factor of 5 greater than that of the largest STZ in the present work. Reference 22 follows Ref. 23 in equating the transformation strain to the universal macroscopic yield strain observed, 0.036 . We suggest that the former is greater than the latter for the following reasons: (a) Physical analogs (Refs. 3 and 4 indicate larger transformation strains, >0.1). (b) This may be explained by the expectation that macroscopic yield involves autocatalytic STZ avalanches, which likely begin at weak spots and for which local strains are higher than the macroscopic strain. ${ }^{12}$ This point is further supported by observations of higher yield stress and strain for smaller dimensions. ${ }^{24}$ When comparing results, one should note that our data were obtained at room temperature, and larger STZs are expected to become active with increasing temperature.

We further reiterate the distinction between data obtained at low strain, when STZs are isolated, and at higher strain, when back stress is lost and STZ interactions with each other are significant. Following Ref. 14, the latter activated flow state is reached for a total volume fraction of $\sim 40 \%$ occupied by STZs, corresponding to macroscopic permanent or anelastic shear strains $>0.033$ for our value of $\gamma_{o}^{T}$. While the present study expands our work into the nonlinear regime, the macroscopic anelastic shear strain is below 0.0060 , with corresponding STZ volume fractions that are still small, $\leq 7.2 \%$. Reported processes such as stress overshoot ${ }^{25,26}$ and loss of neighbors ${ }^{27}$ occur at far higher strains.

In conclusion, a method of constraining samples in bending geometry with bending strains up to 0.0155 was developed. The anelastic strain rate was nonlinear in the applied stress due to the high value of the latter. Combining the constitutive law and zero-bending-moment condition, the nonlinear regime allows us to determine the transformation shear strain and atomic volume of the largest active STZs independently, which are 0.18 and $4.8 \times 10^{-28} \mathrm{~m}^{3}$, respectively. The respective random errors, $1.5 \%$ and $3 \%$, are small because these parameters appear in the exponent in the constitutive law.

\section{ACKNOWLEDGMENTS}

This work was funded by the U.S. National Science Foundation (NSF) (Grant No. DMR- 1708043). The authors are grateful to Dr. F. Pinkerton of General Motors R\&D Center for providing the sample used in this work.

\section{NOMENCLATURE}

"Apparent" strain values below are those determined from

\begin{tabular}{|c|c|}
\hline & $\begin{array}{l}\text { volume fraction of potential STZs comprising } n \\
\text { atoms }\end{array}$ \\
\hline & sample thickness \\
\hline & Young's modulus $\left(=48.2 \mathrm{GPa}^{19}\right)$ \\
\hline$(t=0)$ & $\begin{array}{l}\text { total bending moment immediately after constraint } \\
\text { removal [Eq. (6)] }\end{array}$ \\
\hline$=1, \ldots, 8$ & index denoting spectrum peaks \\
\hline$=13+m$ & $\begin{array}{l}\text { number of atoms an } m \text {-type STZ comprises. Used as } \\
\text { a subscript in Eq. (9) and below }\end{array}$ \\
\hline & mandrel radius \\
\hline & $\begin{array}{l}\text { initial radius of curvature of the sample before } \\
\text { constraint }\end{array}$ \\
\hline & $\begin{array}{l}\text { radius of curvature of the previously constrained } \\
\text { section at time } t\end{array}$ \\
\hline & temperature $(=295.15 \mathrm{~K})$ \\
\hline & $\begin{array}{l}\text { time during unconstrained relaxation after constraint } \\
\text { removal }\end{array}$ \\
\hline & time under constraint \\
\hline & total constraining time, equal to $2 \times 10^{6} \mathrm{~s}$ \\
\hline & distance from sample midplane \\
\hline & malized distance from sam \\
\hline
\end{tabular}
curvature by assuming linear variation across the sample. The term "actual" is used to distinguish strain and stress values from their apparent values.

\section{Greek}

$\bar{\beta}^{2} \quad$ dilatancy factor $\left(\approx 1^{14}\right)$

$\Delta F_{m} \quad$ activation barrier associated with $m$-type STZs

$\Delta F_{21} \quad$ activation free energy for shear transformation of $n=21 \mathrm{STZs}$ 


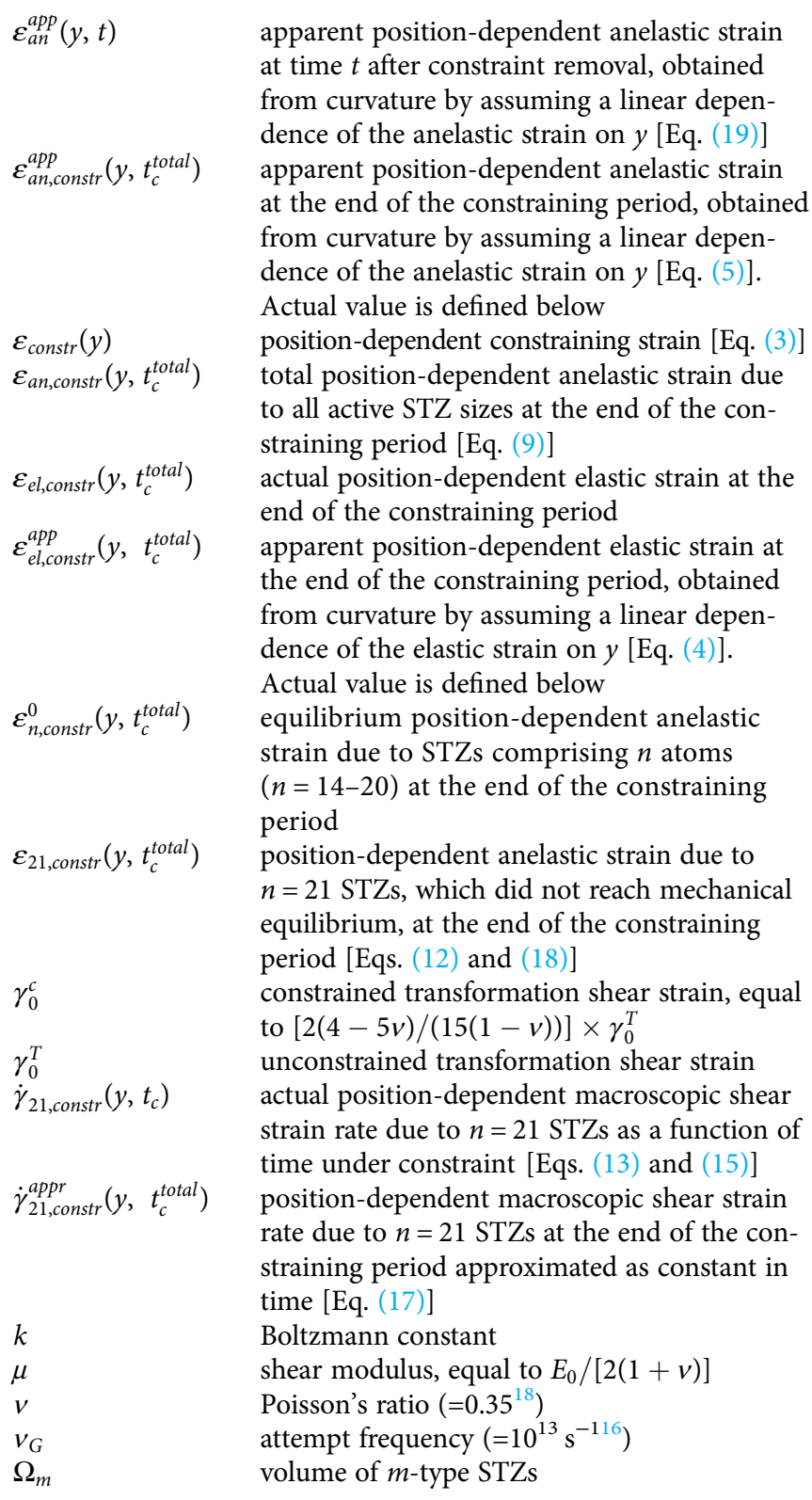

$\Omega_{21}$

$\sigma_{\text {constr }}\left(y, t_{c}^{\text {total }}\right)$

$\sigma_{u}(y, t=0)$

$\overline{\sigma_{S T Z}}$

$\overline{\sigma_{S T Z}} / \mu=0.025^{17}$

$\tau_{n}, n=14, \ldots, 21$

volume of STZs comprising $n=21$ atoms position-dependent applied stress at the end of the constraining period [Eq. (8)] position-dependent unloading stress immediately after constraint removal [Eq. (7)] shear resistance of STZs

time constant for unconstrained anelastic relaxation associated with STZs comprising $n$ atoms

\section{REFERENCES}

${ }^{\mathbf{1}}$ M. F. Ashby and A. L. Greer, Scr. Mater. 54, 321 (2006).

${ }^{2}$ H. J. Leamy, H. S. Chen, and T. T. Wang, Metall. Trans. 3, 699 (1972).

${ }^{3}$ A. S. Argon, Acta Metall. 27, 47 (1979).

${ }^{4}$ P. Schall, D. A. Weitz, and F. Spaepen, Science 318, 1895 (2007).

${ }^{5}$ L. Li, E. R. Homer, and C. A. Schuh, Acta Mater. 61, 3347 (2013).

${ }^{6}$ M. L. Falk and J. S. Langer, Annu. Rev. Condens. Matter Phys. 2, 353 (2011).

${ }^{7}$ M. L. Falk and J. S. Langer, Phys. Rev. E 57, 7192 (1998).

${ }^{8}$ C. A. Schuh, T. C. Hufnagel, and U. Ramamurty, Acta Mater. 55, 4067 (2007).

${ }^{9} \mathrm{E}$. Orowan, in Proceedings of the First U.S. National Congress of Applied Mechanics (ASME, 1952), p. 453.

${ }^{10}$ L. J. Dijkstra and R. J. Sladek, JOM 5, 69 (1953).

"C. Zener, Elasticity and Anelasticity of Metals (University of Chicago Press, Chicago, 1948)

${ }^{12}$ J. D. Ju, D. Jang, A. Nwankpa, and M. Atzmon, J. Appl. Phys. 109, 053522 (2011).

${ }^{13}$ M. Atzmon and J. D. Ju, Phys. Rev. E 90, 042313 (2014).

${ }^{14}$ A. S. Argon and L. T. Shi, Acta Metall. 31, 499 (1983).

${ }^{15} \mathrm{~J}$. D. Eshelby, Proc. R. Soc. A 241, 376 (1957).

${ }^{16}$ A. S. Nowick and B. S. Berry, Anelastic Relaxation in Crystalline Solids (Academic, New York, 1972).

${ }^{17}$ H. Kato, H. Igarashi, and A. Inoue, Mater. Lett. 62, 1592 (2008).

${ }^{18}$ W. H. Wang, Prog. Mater. Sci. 57, 487 (2012).

${ }^{19} \mathrm{~J}$. M. Freitag, R. G. Koknaev, R. Sabet-Sharghi, M. Koknaeva, and Z. Altounian, J. Appl. Phys. 79, 3967 (1996).

${ }^{20}$ B. J. Yang, J. H. Yao, J. Zhang, H. W. Yang, J. Q. Wang, and E. Ma, Scr. Mater. 61, 423 (2009).

${ }^{21}$ J. Das, M. Boström, N. Mattern, Å. Kvick, A. R. Yavari, A. L. Greer, and J. Eckert, Phys. Rev. B 76, 092203 (2007).

22. J. Krausser, K. H. Samwer, and A. Zaccone, Proc. Natl. Acad. Sci. 112, 13762 (2015).

${ }^{23}$ W. L. Johnson and K. Samwer, Phys. Rev. Lett. 95, 195501 (2005).

${ }^{24}$ S. Nachum and A. L. Greer, J. Alloy Compd. 615, S98 (2014).

${ }^{25}$ P. De Hey, J. Sietsma, and A. Van den Beukel, Acta Mater. 46, 5873 (1998).

${ }^{26}$ A. Zaccone, P. Schall, and E. M. Terentjev, Phys. Rev. B 90, 140203(R) (2014).

${ }^{27}$ M. Laurati, P. Maßhoff, K. J. Mutch, S. U. Egelhaaf, and A. Zaccone, Phys. Rev. Lett. 118, 018002 (2017). 